\title{
¿

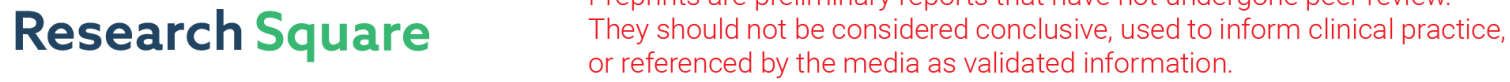 \\ Clean Air Act mitigate the cognitive deterioration in older adults
}

\author{
Yao Yao ( $\square$ yaoyao@nsd.pku.edu.cn ) \\ Peking University https://orcid.org/0000-0002-6723-6152
}

\section{Xiaozhen Lv}

Dementia Care and Research Center, Clinical Research Division, Peking University Institute of Mental Health (Sixth Hospital)

\section{Chengxuan Qiu}

Aging Research Center, Karolinska Institutet

\section{Jiajianghui Li}

Institute of Reproductive and Child Health, Ministry of Health Key Laboratory of Reproductive Health and Department of Epidemiology and Biostatistics, School of Public Health, Peking University

\section{Xiao Wu}

Department of Biostatistics, Harvard T.H. Chan School of Public Health

\section{Hao Zhang}

Department of healthcare policy and research, Weill Cornell Medicine

\section{Dahai Yue}

Department of Health Policy and Management, University of Maryland School of Public Health

\section{Ehab Eshak}

Public Health and Preventive Medicine, Faculty of Medicine, Minia University

\section{Kaarin Anstey}

Neuroscience Research Australia, University of New South Wales

\section{Gill Livingston}

University College London https://orcid.org/0000-0001-6741-5516

\section{Tao Xue}

Institute of Reproductive and Child Health, Ministry of Health Key Laboratory of Reproductive Health and Department of Epidemiology and Biostatistics, School of Public Health, Peking University Jim Zhang

Global Health Institute and the Nicholas School of Environment, Duke University

\section{Huali Wang}

Dementia Care and Research Center, Clinical Research Division, Peking University Institute of Mental Health (Sixth Hospital)

\section{Yi Zeng}

Peking University and Duke University 
Article

Keywords: Clean Air Act, Air pollution, Cognitive functions, Difference-in-differences analysis, Older adults Posted Date: May 18th, 2021

DOI: https://doi.org/10.21203/rs.3.rs-492930/v1

License: (c) (i) This work is licensed under a Creative Commons Attribution 4.0 International License. Read Full License 


\section{Abstract}

Air pollution may accelerate cognitive aging, it is unclear whether large-scale interventions by Clean Air Act can mitigate the cognitive deterioration. Here, we conducted a difference-in-differences analysis based on Chinese Longitudinal Healthy Longevity Survey during 2014 and 2018. Intervention group came from where the government set a strict target of reducing air pollution, whereas control group lived in areas without reduction target. Global cognitive functions were measured using the Mini-Mental State Examination (MMSE). We found the intervention group with implementing Clean Air Act had a significantly smaller decline in MMSE score compared to the control group. Interquartile increases in PM2.5 and SO2 concentrations were significantly associated with a faster decline of MMSE score by 1.78 and 0.92 points, respectively. Implementing stringent clean air policies, especially in low- and middleincome countries may mitigate the risk of cognitive aging in older people.

\section{Main}

Poor cognitive function, a key driver of disability, contributes profoundly to health, social, and economic burdens in the aging society. ${ }^{1}$ Global health care expenditures for dementia reached \$1 trillion in 2018 and are expected to reach a staggering $\$ 2$ trillion by $2030 .{ }^{2,3}$ According to the 2020 Lancet Commission on dementia prevention, 12 modifiable risk factors account for around $40 \%$ of worldwide dementia incidence. Amongst them, air pollution is an emerging risk factor. ${ }^{4}$ Notably, the number of people with dementia is rising more rapidly in low- and middle-income countries (LMICs) than in high-income countries (HICs) due to the population aging and poor environmental factors. ${ }^{5}$

The associations between long-term exposure to fine particulate matter $\left(\mathrm{PM}_{2.5}\right.$; particles $\leq 2.5 \mu \mathrm{m}$ in diameter) and cognitive impairment, dementia, or other neurological disorders have been reported in observational studies. ${ }^{6-15}$ However, a causal inference is hard to be drawn from observational studies due to residential confounding and reverse causation. ${ }^{16,17}$. In this regard, a quasi-experimental study leveraging air quality interventions is advantageous, because an association between changes in a health outcome and changes in air quality brought by an intervention such as clean air policy is more likely to be causal. Such a quasi-experimental design, by mimicking a randomized clinical trial, further strengthen the confidence on putative causality of the similar relationships derived from observational studies. ${ }^{18-20}$

To our knowledge, only one study from the US has estimated the effect of air pollution regulation between 2004 and 2013 on dementia risk. ${ }^{21}$ The long-term effect of the air pollution policy observed in this study, however, may be confounded by time-varying variables such as economic growth, change of lifestyle, and advances in pharmaceuticals and medicines during the long study period ( $>9$ years). Moreover, no study has evaluated the effect of implementation of clean air policy on cognitive health in LMICs.

In late 2013, the most stringent version of China's Clean Air Act (CCAA) was promulgated to address China's widespread and severe air pollution problem. ${ }^{22,23}$ The two waves of the Chinese Longitudinal 
Health Longevity Survey in 2014 and 2018 were aligned with the CCAA implementation plan (from 2014 to 2017). This provides a unique quasi-experiment opportunity to assess the cognitive impact of $\mathrm{PM}_{2.5}$ and co-pollutants. We carried out the present analysis to test the hypothesis that rapid reductions in ambient concentrations of $\mathrm{PM}_{2.5}$ and other pollutants brought by the CCAA implementation would reduce the risk of cognitive deterioration in older adults.

\section{Results}

\section{Descriptive statistics}

The 2812 participants were $81.0 \pm 9.3$ years, comprised of $50.1 \%$ women and $57.6 \%$ rural residents (Table 1). Of all participants, 2251 (79.8\%) were in the intervention group, and the remaining 561 adults were in the control group. Participants in the intervention and control groups were comparable by sex and age. We observed significant between-group differences for rural/urban residence $(P=0.001)$, ethnicity $(P<$ $0.001)$, smoking status $(P=0.02)$ and drinking status $(P=0.04)$. Therefore, we applied inverse probability weighting to control for these differences in our statistical models.

Across all the participants, average MMSE scores declined from 25.8 5.2 in 2014 to $24.0 \pm 7.1$ in 2018 (Table 1). The average reductions of MMSE score were $2.1 \pm 5.8$ and $1.7 \pm 6.5$ among the participants in the control group and the intervention group, respectively. Long-term exposures to (annual concentrations of) $\mathrm{PM}_{2.5}, \mathrm{PM}_{10}, \mathrm{SO}_{2}, \mathrm{NO}_{2}$, and $\mathrm{CO}$ across all the participants were 58.2, 84.2, 25.5, 26.6, and 0.856 $\mu \mathrm{g} / \mathrm{m}^{3}$, respectively in 2014 . The exposures in 2018 were reduced by $28.7 \%, 16.6 \%, 49.8 \%, 4.1 \%$, and $9.3 \%$, respectively. In contrast, long-term $\mathrm{O}_{3}$ exposures in 2018 was increased by $9.5 \%$ from $85.5 \mu \mathrm{g} / \mathrm{m}^{3}$ in 2014 to $94.5 \mu \mathrm{g} / \mathrm{m}^{3}$ in 2018. $\mathrm{PM}_{2.5}, \mathrm{PM}_{10}$, and $\mathrm{SO}_{2}$ showed a greater reduction in the intervention group than in the control group (Supplementary Figure A2).

\section{Effect of the CCAA intervention on cognitive function}

The intervention effects on MMSE score, estimated in models with different covariates, are shown in Figure 1. When adjusted for no covariates (Model 1), the intervention group had a smaller decline in MMSE score than the control group, showing that the CCAA implementation was associated with an incremental MMSE score of 1.36 (95\% confidence interval [Cl]: $0.47,2.25)$ points. In the fully-adjusted model, this estimate on the score improvement was larger $(2.45$ with $95 \% \mathrm{Cl}$ of $1.32,3.57)$. In a sensitivity analysis by excluding the IPW weights and the offset of MMSE trend, the estimates were generally consistent to the fully-adjusted model results (Supplementary Figure A3). Moreover, statistical significances for intervention effects were consistently robust when the $\geq 5 \%$ reduction target was replaced with three larger reduction targets. The sample size of the control/intervention group using the three stricter cut-off points were 1027/1785, 1223/1589, 1800/1012, respectively. The estimate effect on improvement of MMSE score was slightly lower in the high cut-off group compared with low cut-off group. This phenomenon might be explained by the reason that more and more provinces with actually 
moderate emission reduction target were assigned to the control group as the threshold went up, thus making the difference of CCAA policy strength between the two groups become smaller.

We also examined potential effect modifications (Supplementary Figure A4). We found the effect estimates were potentially modified by age, ethnicity, education, marital status, or physical activity ( $P$ for intervention-covariate interaction $<0.05)$. We found that the CCAA beneficial effect was amplified by a higher education level, being married, being less old, or being physically more active. The beneficial effect estimate was smaller in participants of Han ethnicity. This might be due to ethnicity differences in genetics or lifestyle characteristics. However, this needs to be interpreted with caution, given the small number of non-Han participants $(n=189 ; 6.72 \%)$ in our cohort.

\section{Associations between changes in pollutant exposure and cognitive function decline}

Figure 2 shows MMSE score changes associated with per unit increase in pollutant exposure, adjusting for the selected covariates. Separate cross-sectional analyses of the 2014 wave data and the 2018 wave data generated similar results for all the pollutants except $\mathrm{O}_{3}$, indicating increasing exposure was associated with decreasing MMSE score. According to the DID analysis, increasing exposure to $\mathrm{PM}_{2.5}$ or to $\mathrm{SO}_{2}$ was significantly associated with faster cognitive decline. Comparing the results from the DID analysis and those from the cross-sectional analyses, we found the $\mathrm{PM}_{2.5}$ effect was the most robust, as the separate 2014 and 2018 wave cross-sectional analyses both found a significant MMSE association with $\mathrm{PM}_{2.5}$. Not surprisingly, due to a strong inverse correlation between $\mathrm{PM}_{2.5}$ and $\mathrm{O}_{3}$, we observed a positive association between $\mathrm{O}_{3}$ and MMSE score in the DID analysis. Adjusting for ambient temperature generated did not change these the associations (Supplementary Figure A5). Additionally, we conducted DID analyses in subgroups stratified by age, sex, ethnicity, rural/urban residence, marital status, and years of schooling. We found the effect modification similar to that found in the intervention analysis described above (Supplementary Figure A6). Finally, we also associated pollutant exposure changes with MMSE score changes using nonlinear models (Figure 3 ), which yielded results comparable to the effects observed in the linear models. The nonlinear models consistently showed an inverse association of MMSE score with $\mathrm{PM}_{2.5}$ or $\mathrm{SO}_{2}$, and a positive association with $\mathrm{O}_{3}$. In a sensitivity analysis by linking MMSE scores with $\mathrm{PM}_{2.5}$ and $\mathrm{O}_{3}$ changes jointly, the estimate for $\mathrm{O}_{3}$ was statistically non-significant while conditioning on the $\mathrm{PM}_{2.5}$ level (Supplementary Figure A7). The two pollutant models also suggested $\mathrm{SO}_{2}$ has a significant impact on MMSE change after controlling $\mathrm{O}_{3}$ or $\mathrm{PM}_{2.5}$ on a constant level. However, the 2018 wave cross-sectional analysis did not find a significant MMSE association with $\mathrm{SO}_{2}$ exposure. It is possible that the $\mathrm{SO}_{2}$ effect observed in the DID model reflects the simultaneous

reductions of both pollutants by controlling coal combustion, a major source of both $\mathrm{PM}_{2.5}$ and $\mathrm{SO}_{2} \cdot{ }^{39}$

\section{Discussion}

In this quasi-experimental study in a nationwide sample of older adults in China, we observed a beneficial effect of a national clean air policy (the CCAA implementation) on cognitive function. Specifically, the 
covariates-adjusted mean MMSE score decline from 2014 to 2018 was 2.45 points (95\% Cl: 1.32, 3.57) less in the policy intervention group than in control group of participants whose annual $\mathrm{PM}_{2.5}$ exposure reduction was $<5 \%$. Using annual average ambient pollutant concentrations at the residence level as exposure estimates, we found that increased $\mathrm{PM}_{2.5}$ exposure was associated with decreased MMSE score across all participants in both the 2014 and 2018 waves of the survey. More importantly, we found that smaller MMSE score reductions from 2014 to 2018 were associated with greater $\mathrm{PM}_{2.5}$ reductions over the same period. Taken together, these findings support a potential causal role of $\mathrm{PM}_{2.5}$ in accelerating cognitive decline in older adults, contributing to the emerging evidence, reported in previous observational studies, that long-term $\mathrm{PM}_{2.5}$ exposure is a risk factor of cognitive function. ${ }^{6-15}$

This study is the first to assess the effectiveness of implementing a national clean air policy in attenuating aging-driven cognitive. By integrating the CCAA implementation targets for $\mathrm{PM}_{2.5}$ between 2014 to 2017 with the CLHLS surveys in 2014 (before the implementation) and 2018 (after the implementation), we had the unique opportunity to design this quasi-experimental study in which we evaluated the effect of the clean air policy targeted on $\mathrm{PM}_{2.5}$ reduction on cognitive function. In our difference-in-differences (DID) analysis approach, we further leveraged an experimental design consisting of an intervention group and control group based on differences in $\mathrm{PM}_{2.5}$ reduction targets. This allowed us to obtain an appropriate counterfactual framework to estimate a causal effect. A strength of the DID approach is that through comparing same individual to itself at different time points, thus potential confounders such as genetic factors and lifestyles are controlled. Similar to previous DID designs, we controlled for certain slow-changing individual-level variables such as socio-demographics, lifestyles, and socioeconomic status. ${ }^{17,19}$ We also controlled for annual temperature that may have varied with time. These adjustments further reduced potential confounding to the effects of the CCAA intervention and $\mathrm{PM}_{2.5}$ observed in our study.

The DID analysis approach has been used in environmental interventional studies of other health outcomes. ${ }^{18}$ Previous studies have focused primarily on PM-related mortality. ${ }^{16,17,19,40,41}$ In addition, our previously published works found that Clean Air Actions in China was associated with decreased household medical expenditures ${ }^{42}$ and reduced prevalence of depressive symptoms. ${ }^{43}$ To date, however, only one study in the United State has assessed the impact of the air pollution regulations on dementia diagnosis in people older than 75 years. ${ }^{21}$ However, that study was a population-level analysis of the association between reductions in annual average $\mathrm{PM}_{2.5}$ concentration from 2004 to 2013 and reductions in dementia incidence rate. Unlike the present study, that US based study did not control individual-level data such as lifestyle, socioeconomic status, health conditions, and other individual-level statuses. These individual-level variables may have changed substantially during the long evaluation period (2004-2013), which may confound the effect of the policy. In the present study, we conducted a series of sensitivity analyses considering individual-level variables and their changes, confirming the robustness of the significant beneficial effect on cognitive function of $\mathrm{PM}_{2.5}$ exposure reduction brought by the CCAA implementation. 
Our finding from the present study is consistent with previous epidemiologic findings on the detrimental effects of air pollutants on brain health. ${ }^{6-15}$ A meta-analysis of 31 studies, including both cross-sectional and longitudinal analyses, provided compelling evidence that air pollution exposure is associated with accelerated cognitive decline across the life course. ${ }^{29}$ The present study, through a study design mimicking a randomized clinical trial, further strengthens the confidence on putative causality of similar conclusions from existing observational studies. ${ }^{20}$ These epidemiologic findings are supported by biological mechanisms by which fine particle matter impairs cognitive function. Experimental research has shown that fine and especially ultrafine particles (both are components of $\mathrm{PM}_{2.5}$ ) can enter the brain either via circulation or intra-nasally by direct translocation through the olfactory bulb, leading to detrimental and toxic effects in the brain. ${ }^{44-46}$ Several animal studies have shown increased neural inflammation in response to $\mathrm{PM}_{2.5}$ exposures. ${ }^{47-49}$ In a post-mortem study of 19 humans aged 34 to 83 years, who had died of non-neurologic causes, expression of cyclooxygenase-2 (COX-2), an inflammatory mediator, and accumulation of the 42-amino acid form of $\beta$-amyloid (Aß42), a cause of neuronal dysfunction, were higher among those who had lived in severely polluted cities in Mexico than among those who had lived in less polluted cities. ${ }^{50} \mathrm{~A}$ recent study in humans showed an association between air pollution and local gyrification index, a marker of local brain atrophy in the aging brain, suggesting that chronic exposure to $\mathrm{PM}_{2.5}$ may influence the physiological aging process of the brain. ${ }^{51}$

A major strength of this study is the use of the quasi-experimental study design to examine the potential causal link between CCAA policy and cognitive decline, by taking advantage of the rapid reduction of air pollution over a relatively short time window (4 years). The causal inference is strengthened by including a counterfactual analysis feature, i.e., the intervention group of participants who have experienced a $\mathrm{PM}_{2.5}$ reduction exceeding a target (e.g., $5 \%$ annually) in comparison with the control group of participants had not experienced a target reduction in $\mathrm{PM}_{2.5}$ exposure. A previous study has shown that about $90 \%$ of $\mathrm{PM}_{2.5}$ variations during 2014 and 2017 in China were attributable to the CCAA implementation. ${ }^{23}$ Therefore, $\mathrm{PM}_{2.5}$ reduction could serve as a discriminative indicator for the CCAA effectiveness in our DID models. Indeed, the significant intervention effect based on group comparison is further strengthened by significant "exposure-response" relationship between MMSE score change with $\mathrm{PM}_{2.5}$ change from 2014 to 2018 . Another strength is our ability to use residence-level air pollution estimates (as opposed to city-level or ZIP-code-level widely used in previous studies) and individual-level socio-demographic and behavioral data. Moreover, we conducted a nationwide, prospective cohort study with neuropsychological data and individual characteristics in a diverse population in China, increasing the generalizability of our study findings. As China is one of the largest LMICs in the world, our findings may have policy implications for dementia prevention among LMICs.

This study has some limitations. First, its quasi-experimental design does not eliminate the possibility of residual confounding. Although we found improvement in cognitive decline after implementing the CCAA policy, we cannot rule out the possibility that there may be some other changes during this period of time or as a result of the policy (e.g., more physical activity or social participation after the air quality 
improves) which caused the slowing down of cognitive function decline. However, our methodology, including use of time and policy fixed effects as well a time varying variable, helped mitigate this limitation. Second, model-based residence-level exposure estimates cannot be verified with measured data for all areas, although the models have been well validated. ${ }^{52}$ Finally, the participants in our control group included those living in the areas that had been in poorer provinces with a range of other risk factors, such as cigarette smoking, and poverty. We used sensitivity analyses to evaluate the impact of some of these potential confounding factors.

Findings from this study have notable public health policy implications in the aging society. Dementia burden is projected to rapidly increase globally, especially in LMICs. Controlling any preventable risk factors is of paramount importance for a healthy aging society. The evidence generated in the presented study, with a unique strength of causal inference, support the need to implement well-designed clean air policies to reduce population and individual exposure to air pollutants especially $\mathrm{PM}_{2.5}$. The need is particularly urgent considering the already-known large cardio-respiratory disease burden of air pollution. ${ }^{53}$ The policy-oriented interventions offer widespread protection and enhanced health with less reliance on conventional individual-centered lifestyle modifications. ${ }^{54}$

In conclusion, this quasi-experimental study adds new evidence of supporting causal inference that $\mathrm{PM}_{2.5}$ exposure accelerates cognitive aging in older people. Considering the rapid population aging and increasing global disease burden from dementia, implementing policy-oriented interventions such as clean air policies, especially in LMICs, are of paramount importance for heathy aging.

\section{Methods}

This study was conducted in accordance with the Strengthening the Reporting of Observational Studies in Epidemiology (STROBE) guideline. The STROBE checklist can be found as part of the Supplementary Information.

\section{Study design and participants}

We used data from the Chinese Longitudinal Healthy Longevity Survey (CLHLS), a prospective cohort study on the determinants of healthy aging and longevity among older population ( $\geq 60$ years) in China. Briefly, the CLHLS is a nationwide survey on a randomly selected sample from half of the counties and cities in 23 of the 31 provinces, covering about $85 \%$ of the total population of China (Figure 4). Beginning in 1998, a new wave of survey has been conducted every 2-3 years. To reduce attrition in numbers from death and loss to follow-up, new 'make-up' participants are enrolled during each follow-up wave. Of note, the CLHLS oversampled male octogenarians, nonagenarians, and centenarians, further shaping the age distribution of the analytic sample. ${ }^{24}$ Trained interviewers conducted the surveys at participants' homes following a structured questionnaire. They collected data on sociodemographic characteristics, lifestyle, cognitive function, psychological status, and physical capacity. More details on the sampling procedure and assessment of data quality can be found in previous publications. ${ }^{25,26}$ 


\section{The intervention and exposure assessment}

This interventional study was based on the effectiveness in $\mathrm{PM}_{2.5}$ reduction through the implementation of China's Clean Air Act (CCAA) by provincial governments. We designated CLHLS participants who lived in provinces where the government set a target of reducing $\mathrm{PM}_{2.5}$ concentrations $\geq 5 \%$ annually from 2014 onward into the intervention group and the rest of the participants into the control group (Figure 4). We identified 18 provinces with and 5 provinces without an established target of $\mathrm{PM}_{2.5}$ reduction in 2014 (Supplementary Table A2). Description of CCAA was presented in Supplementary Methods, China's Clean Air Act.

We generated residence-specific ambient concentrations of six pollutants $\left(\mathrm{PM}_{2.5}, \mathrm{PM}_{10}, \mathrm{SO}_{2}, \mathrm{NO}_{2}, \mathrm{O}_{3}\right.$, and CO) using a previously published method. ${ }^{27}$ Briefly, we coupled the original monthly-average concentration data with participants survey dates and residential addresses to calculate the average concentrations of the six air pollutants during the 12 months preceding the survey dates. These annual average concentrations were regarded as long-term exposure estimates. We also obtained participantspecific estimates of land surface temperature from a weather-forecast research (WRF) model. ${ }^{28}$ Detailed descriptions of air pollution and weather assessment are presented in Supplementary Methods, Environmental Assessment.

\section{Health outcomes and covariates}

The primary outcome was global cognitive function assessed using the Mini-Mental State Examination (MMSE), a screening tool for dementia. The MMSE has been frequently used to track changes in cognitive function over time, as this tool can reliably detect cognitive deterioration. ${ }^{29}$ This tool is especially useful for people unable to go through complex clinical diagnostic testing. ${ }^{30}$ The global score of MMSE ranges from 0 to 30, with a higher score indicating a better cognitive function. ${ }^{31}$ We calculated cognitive decline by subtracting participants' MMSE score in 2014 from their scores in 2018. Detailed description and scoring methods on MMSE were showed in Supplementary Methods, Mini-Mental State Examination.

We assessed a range of demographic, behavioral, and socioeconomic factors as covariates, including age, sex, education, marital status, ethnicity, rural/urban residence, occupation before retirement, survey month, alcohol drinking, smoking, physical activity, fruit intake, vegetable intake, water quality, living condition, and income source. Participants were encouraged to answer as many questions as possible. No proxy was used for objective questions such as assessment of cognitive function and physical performance. ${ }^{24}$ The detailed definition of covariates can be seen in the Supplementary Methods, Descriptions of covariates. In addition, we included model-estimated ambient temperature (see above) as a covariate.

\section{Statistical analyses}


We calculated within-individual differences between the MMSE scores assessed in 2014 and 2018 . We used mixed-effect model in which within-individual MMSE difference was dependent variable, intervention group and selected covariates were fixed-effect independent variables (see below), and participant ID was a random-effect variable. We used between-group difference in within-individual MMSE score difference to interpret the impact of the intervention (CCAA).

The comparability between the intervention group and the control group may be undermined due to the differences in aspects of (1) baseline demographic characteristics, (2) long-term trend of outcome variables, and (3) changes in longitudinal risk factors during the study period. To control for those differences, firstly, we applied inverse probability weighting (IPW) to match up the two groups in terms of baseline population characteristics including age, education, sex, residence, ethnicity, occupation before retirement, alcohol drinking status, smoking status, and living condition. ${ }^{32,33}$ Secondly, we utilized withinindividual MMSE score changes from another prior wave of survey (in 2011) to the 2014 wave as a variable to approximate the longer-term individual-level MMSE score trends. We did not use the difference of MMSE score between 2018 and 2014 minus the difference between 2014 and 2011 as the dependent variable, because it may introduce some confounding factors that change with time as it covers a relatively long time-span. Thirdly, we further adjusted for temporal changes in the longitudinal risk factors for cognitive change, including survey month, marital status, alcohol drinking, smoking, physical activity, fruit intake, vegetable intake, water quality, living condition, and income source.

To assess the relationship between MMSE score and pollutant exposure, we conducted cross-sectional analyses of the 2014 and 2018 wave data, respectively, using linear regression models controlling for selected covariates. Furthermore, we used Difference-in-Differences (DID) analysis to assess the MMSE score change in association with pollutant exposure change from 2014 to 2018 . The model is described as:

$$
\mathrm{MMSE}_{i, t} \sim \beta \mathrm{x}_{i, t}+\gamma z_{i, t}+\eta_{i}+\Delta t \ldots \ldots \text { (1) }
$$

where $i$ denotes subject index; $t$ denotes index of CLHLS wave and equals to 0 ( $7^{\text {th }}$ wave) or 1 ( $8^{\text {th }}$ wave); $\mathrm{MMSE}_{i, t}$ denotes MMSE score for the $i^{\text {th }}$ subject at the wave $t, x_{i, t}$ denotes air pollutant exposure; $\beta$ is the change in MMSE per unit change in exposure; $z_{i, t}$ denotes the selected covariates described above; $\gamma$ is the coefficient for a specific covariate; $\eta_{i}$ denotes the subject-specific fixed effect; and $\Delta t$ denotes the effect of temporal trend. To control for the seasonal variation in MMSE, we included an index of survey month in the main models. We also used an alternative approach by replacing the survey month with a spline term of monthly temperature (degrees of freedom $=3$ ).

\section{Sensitivity and effect modification analyses}

First, we analyzed whether cognitive decline and $\mathrm{PM}_{2.5}$ exposure had a different linear trend in intervention group versus the control group before the 2014 CCAA implementation using the 2011 and 2014 waves of CLHLS dataset (Supplementary Figure A1). Second, we applied different stricter cut-offs 
of the PM reduction targets $(10 \%, 12 \%$, and $15 \%)$, e.g., participants who lived in the places where the government set a target of reducing $\mathrm{PM}_{2.5}$ concentrations of more than $10 \%$ as the intervention group and the rest of the participants were assigned to the control group Third, we repeated the analysis without IPW weights and without offset of longer-term MMSE trend. Fourth, we examined the interaction between pollutant exposure and intervention group indicator to assess whether exposure-outcome relationships were different between the two groups. Fifth, we explored the nonlinear exposure-response association through replacing the linear term of exposure by its spline expansions. Finally, we derived two-pollutant nonlinear models through parametrizing the exposure term using a two-dimensional spline function. The two-pollutant exposure-response associations were utilized to explore how temporal variation in a pollutant was linked with the MMSE score change, given the scenario where the other modelled pollutant was unchanged (Supplementary Methods, Two-pollutant exposure-response analysis).

All statistical analyses were performed using R (version 3.3.2; R Foundation for Statistical Computing, Vienna, Austria). Imputation was performed using the mice package. ${ }^{34}$ The linear mixed-effects and fixed-effects models were inferred using the Ime 4 package and the $p / m$ package, ${ }^{35}$ respectively. Inverse probability weights were calculated using the ipw package. ${ }^{36}$ Penalized spline functions were parameterized using the $m g c v$ package ${ }^{37}$ and inference of the nonlinear mixed-effects models was done using the gamm4 package. ${ }^{38}$

\section{Declarations}

\section{Data availability}

The data that support the findings of this study are available from Center for Healthy Aging and Development Studies, Peking University but restrictions apply to the availability of residential data, which were used under license for the current study, and so are not publicly available. Data are however available from the authors upon reasonable request and with permission of Prof Yi Zeng (zengyi@nsd.pku.edu.cn).

\section{Competing interests}

The authors declare that they have no competing interests.

\section{Funding}

This work was supported by the National Key R\&D Program of China (2018YFC2000400, 2017YFC1311100), National Natural Sciences Foundation of China (81903392, 81941021), China Postdoctoral Science Foundation funded project (2019M650359), the U.S. National Institute on Aging, National Institutes of Health (P01AG031719), the Duke/Duke-NUS Collaboration Pilot Project, and PKUBaidu Fund.

\section{Contributions}


Y.Y., X.T., Z.J., W.H. and Z.Y. designed the research and directed its implication; Y.Y., and X.T. prepared and analyzed the data; Y.Y., L.X., and X.T. drafted the manuscript; all co-authors revised the manuscript.

\section{Acknowledgements}

We are grateful to the CLHLS participants for providing the data for this research. The CLHLS was supported by funds from the U.S. National Institute on Aging, National Institutes of Health, the Duke/Duke-NUS Collaboration Pilot Project, the National Natural Science Foundation of China, the China Social Science Foundation and the United Nations Fund for Population Activities (UNFPA). The CLHLS was managed by the Center for Healthy Aging and Development Studies, Peking University. We also thank the support from the Healthy Aging Consortium of the China Cohort Consortium (see http://chinacohort.bjmu.edu.cn/).

We thank Prof. Hongkai Li (Shandong University), Dr. Luke Parsons (Duke University), Prof. Keyang Liu (Osaka University), Dr. Lei Hou (Shandong University), and Dr. Bin Han (University of Washington, Seattle) for their thoughtful comments during revising and improving this paper.

\section{References}

1 Prince, M. et al. The global prevalence of dementia: a systematic review and metaanalysis. Alzheimers Dement 9, 63-75 e62, doi:10.1016/j.jalz.2012.11.007 (2013).

2 Prince, M. et al. World Alzheimer report 2015: The Global Impact of Dementia: An analysis of prevalence, incidence, cost and trends. (2015).

3 Wimo, A. et al. The worldwide costs of dementia 2015 and comparisons with 2010. Alzheimers Dement 13, 1-7, doi:10.1016/j.jalz.2016.07.150 (2017).

4 Livingston, G. et al. Dementia prevention, intervention, and care: 2020 report of the Lancet Commission. Lancet 396, 413-446, doi:10.1016/S0140-6736(20)30367-6 (2020).

5 Collaborators, G. B. D. R. F. Global, regional, and national comparative risk assessment of 84 behavioural, environmental and occupational, and metabolic risks or clusters of risks for 195 countries and territories, 1990-2017: a systematic analysis for the Global Burden of Disease Study 2017. Lancet 392, 1923-1994, doi:10.1016/S0140-6736(18)32225-6 (2018).

6 Zhang, X., Chen, X. \& Zhang, X. The impact of exposure to air pollution on cognitive performance. Proc Natl Acad Sci U S A 115, 9193-9197, doi:10.1073/pnas.1809474115 (2018).

7 Schikowski, T. \& Altug, H. The role of air pollution in cognitive impairment and decline. Neurochem Int 136, 104708, doi:10.1016/j.neuint.2020.104708 (2020).

8 Kulick, E. R. et al. Long-term exposure to ambient air pollution, APOE-epsilon4 status, and cognitive decline in a cohort of older adults in northern Manhattan. Environ Int 136, 105440, 
doi:10.1016/j.envint.2019.105440 (2020).

$9 \quad$ Kulick, E. R. et al. Long-term exposure to air pollution and trajectories of cognitive decline among older adults. Neurology 94, e1782-e1792, doi:10.1212/WNL.0000000000009314 (2020).

10 Tzivian, L. et al. Long-Term Air Pollution and Traffic Noise Exposures and Mild Cognitive Impairment in Older Adults: A Cross-Sectional Analysis of the Heinz Nixdorf Recall Study. Environ Health Perspect 124, 1361-1368, doi:10.1289/ehp.1509824 (2016).

11 Weuve, J. et al. Exposure to particulate air pollution and cognitive decline in older women. Arch Intern Med 172, 219-227, doi:10.1001/archinternmed.2011.683 (2012).

12 Grande, G., Ljungman, P. L. S., Eneroth, K., Bellander, T. \& Rizzuto, D. Association Between Cardiovascular Disease and Long-term Exposure to Air Pollution With the Risk of Dementia. JAMA Neurol 77, 801-809, doi:10.1001/jamaneurol.2019.4914 (2020).

13 Tallon, L. A., Manjourides, J., Pun, V. C., Salhi, C. \& Suh, H. Cognitive impacts of ambient air pollution in the National Social Health and Aging Project (NSHAP) cohort. Environ Int 104, 102-109, doi:10.1016/j.envint.2017.03.019 (2017).

14 Shi, L. et al. Long-term effects of PM(2.5) on neurological disorders in the American Medicare population: a longitudinal cohort study. The Lancet. Planetary health, doi:10.1016/s2542-5196(20)302278 (2020).

15 Wang, J. et al. Fine Particulate Matter and Poor Cognitive Function among Chinese Older Adults: Evidence from a Community-Based, 12-Year Prospective Cohort Study. Environmental health perspectives 128, 67013, doi:10.1289/ehp5304 (2020).

16 Wu, X., Braun, D., Schwartz, J., Kioumourtzoglou, M. \& Dominici, F. J. S. A. Evaluating the impact of long-term exposure to fine particulate matter on mortality among the elderly. Science Advances 6 , eaba5692 (2020).

17 Yu, W., Guo, Y., Shi, L. \& Li, S. The association between long-term exposure to low-level PM2.5 and mortality in the state of Queensland, Australia: A modelling study with the difference-in-differences approach. PLoS Med 17, e1003141, doi:10.1371/journal.pmed.1003141 (2020).

18 Bind, M. A. Causal Modeling in Environmental Health. Annu Rev Public Health 40, 23-43, doi:10.1146/annurev-publhealth-040218-044048 (2019).

19 Wang, Y. et al. Estimating Causal Effects of Long-Term PM2.5 Exposure on Mortality in New Jersey. Environ Health Perspect 124, 1182-1188, doi:10.1289/ehp.1409671 (2016).

20 Goldman, G. T. \& Dominici, F. Don't abandon evidence and process on air pollution policy. Science 363, 1398-1400, doi:10.1126/science.aaw9460 (2019). 
21 Bishop, K. C., Ketcham, J. D. \& Kuminoff, N. V. Hazed and confused: the effect of air pollution on dementia. Report No. 0898-2937, (National Bureau of Economic Research, 2018).

22 China, S. C. o. t. P. s. R. o. Notice of the general office of the state council on issuing the air pollution prevention and control action plan. (2013).

23 Zhang, Q. et al. Drivers of improved PM2.5 air quality in China from 2013 to 2017. Proc Natl Acad Sci U S A 116, 24463-24469, doi:10.1073/pnas.1907956116 (2019).

24 Zeng, Y., Poston, D. L., Vlosky, D. A. \& Gu, D. Healthy longevity in China: demographic, socioeconomic, and psychological dimensions. Vol. 20 (Springer Science \& Business Media, 2008).

25 Zeng, Y., Feng, Q., Hesketh, T., Christensen, K. \& Vaupel, J. W. Survival, disabilities in activities of daily living, and physical and cognitive functioning among the oldest-old in China: a cohort study. Lancet 389, 1619-1629, doi:10.1016/S0140-6736(17)30548-2 (2017).

26 Zeng, Y. Toward deeper research and better policy for healthy aging - using the unique data of Chinese longitudinal healthy longevity survey. China Economic Journal 5, 131-149, doi:10.1080/17538963.2013.764677 (2012).

27 Xiao T, Lei K, Jiang Z, et al. Data From:A Six-year long High-resolution Air Quality Reanalysis Dataset over China from 2013 to 2018. In: Science Data Bank; 2020.

28 Xue, T., Zhu, T., Zheng, Y. X. \& Zhang, Q. Declines in mental health associated with air pollution and temperature variability in China. Nat Commun 10, doi:ARTN 216510.1038/s41467-019-10196-y (2019).

29 Clifford, A., Lang, L., Chen, R., Anstey, K. J. \& Seaton, A. Exposure to air pollution and cognitive functioning across the life course--A systematic literature review. Environ Res 147, 383-398, doi:10.1016/j.envres.2016.01.018 (2016).

30 Pezzotti, P., Scalmana, S., Mastromattei, A., Di Lallo, D. \& Progetto Alzheimer Working, G. The accuracy of the MMSE in detecting cognitive impairment when administered by general practitioners: a prospective observational study. BMC Fam Pract 9, 29, doi:10.1186/1471-2296-9-29 (2008).

31 Cummings, J. L. Mini-Mental State Examination: Norms, Normals, and Numbers. JAMA 269, 2420-2421, doi:10.1001/jama.1993.03500180112046 \%J JAMA (1993).

32 Abadie, A. Semiparametric Difference-in-Differences Estimators. The Review of Economic Studies 72, 1-19, doi:10.1111/0034-6527.00321 \%J The Review of Economic Studies (2005).

33 Mansournia, M. A. \& Altman, D. G. Inverse probability weighting. BMJ 352, i189, doi:10.1136/bmj.i189 (2016). 
34 Buuren, S. v. \& Groothuis-Oudshoorn, K. J. J. o. s. s. mice: Multivariate imputation by chained equations in R. 1-68 (2010).

35 Croissant Y, Millo GJJoss. Panel data econometrics in R: The plm package. 2008;27(2):1-43.

36 van der Wal, W. M. \& Geskus, R. B. J. J. S. S. ipw: an R package for inverse probability weighting. 43, 1-23 (2011).

37 Wood, S. \& Wood, M. S. J. R. p. v. Package 'mgcv'. 1, 29 (2015).

38 Wood S, Scheipl F. gamm4: Generalized additive mixed models using mgcv and Ime4. R package version 0.2-3. In: US Environmental Protection Agency; 2014.

$39 \mathrm{Ku}, \mathrm{T}$. et al. Synergistic effects of particulate matter $(\mathrm{PM}(2.5))$ and sulfur dioxide (SO(2)) on neurodegeneration via the microRNA-mediated regulation of tau phosphorylation. Toxicology research 6 , 7-16, doi:10.1039/c6tx00314a (2017).

40 Xue, T. et al. Change in the number of PM2.5-attributed deaths in China from 2000 to 2010: Comparison between estimations from census-based epidemiology and pre-established exposureresponse functions. Environ Int 129, 430-437, doi:10.1016/j.envint.2019.05.067 (2019).

41 Leogrande, S. et al. Industrial air pollution and mortality in the Taranto area, Southern Italy: A difference-in-differences approach. Environ Int 132, 105030, doi:10.1016/j.envint.2019.105030 (2019).

42 Xue, T. et al. Clean air actions in China, PM2.5 exposure, and household medical expenditures: A quasi-experimental study. PLoS Med 18, e1003480, doi:10.1371/journal.pmed.1003480 (2021).

$43 \quad$ Xue, T. et al. Long-term PM2. 5 exposure and depressive symptoms in China: a quasiexperimental study. The Lancet Regional Health-Western Pacific 6, 100079 (2020).

44 Oberdorster, G. \& Utell, M. J. Ultrafine particles in the urban air: to the respiratory tract-and beyond? Environ Health Perspect 110, A440-441, doi:10.1289/ehp.110-1240959 (2002).

45 Oberdorster, G. et al. Translocation of inhaled ultrafine particles to the brain. Inhal Toxicol 16, 437-445, doi:10.1080/08958370490439597 (2004).

46 Elder, A. et al. Translocation of inhaled ultrafine manganese oxide particles to the central nervous system. Environ Health Perspect 114, 1172-1178, doi:10.1289/ehp.9030 (2006).

47 Campbell, A. et al. Particulate matter in polluted air may increase biomarkers of inflammation in mouse brain. Neurotoxicology 26, 133-140, doi:10.1016/j.neuro.2004.08.003 (2005).

48 Levesque, S. et al. Diesel exhaust activates and primes microglia: air pollution, neuroinflammation, and regulation of dopaminergic neurotoxicity. Environ Health Perspect 119, 11491155, doi:10.1289/ehp.1002986 (2011). 
49 Bai, K. J. et al. Microglial activation and inflammation caused by traffic-related particulate matter. Chem Biol Interact 311, 108762, doi:10.1016/j.cbi.2019.108762 (2019).

50 Calderon-Garciduenas, L. et al. Brain inflammation and Alzheimer's-like pathology in individuals exposed to severe air pollution. Toxicol Pathol 32, 650-658, doi:10.1080/01926230490520232 (2004).

51 Nussbaum, R. et al. Associations of Air Pollution and Noise with Local Brain Structure in a Cohort of Older Adults. Environ Health Perspect 128, 67012, doi:10.1289/EHP5859 (2020).

52 Huang, Y. L. \& Batterman, S. Residence location as a measure of environmental exposure: a review of air pollution epidemiology studies. Journal of exposure analysis and environmental epidemiology 10, 66-85, doi:10.1038/sj.jea.7500074 (2000).

53 Landrigan, P. J. et al. The Lancet Commission on pollution and health. Lancet (London, England) 391, 462-512, doi:10.1016/s0140-6736(17)32345-0 (2018).

54 Susser, M. \& Susser, E. Choosing a future for epidemiology: I. Eras and paradigms. Am J Public Health 86, 668-673, doi:10.2105/ajph.86.5.668 (1996).

\section{Tables}

Table 1. Characteristics of study participants. 


\begin{tabular}{|c|c|c|c|c|c|c|}
\hline \multirow[t]{2}{*}{ Variable } & \multicolumn{2}{|c|}{ Total sample $(\mathrm{n}=2812)$} & \multicolumn{2}{|c|}{ Control group $(\mathrm{n}=561)$} & \multicolumn{2}{|c|}{$\begin{array}{l}\text { Intervention group } \\
(\mathrm{n}=2251)\end{array}$} \\
\hline & 2014 & 2018 & 2014 & 2018 & 2014 & 2018 \\
\hline Age (year) & $81.0(9.3)$ & -- & $81.4(9.7)$ & -- & $80.9(9.2)$ & -- \\
\hline Education (year) & $2.8(3.6)$ & -- & $2.9(3.8)$ & - * & $2.8(3.6)$ & -- \\
\hline Female, n (\%) & $\begin{array}{c}1408 \\
(50.1 \%)\end{array}$ & -- $^{*}$ & $\begin{array}{c}294 \\
(52.4 \%)\end{array}$ & $-{ }^{*}$ & $\begin{array}{c}1114 \\
(49.5 \%)\end{array}$ & -- $^{*}$ \\
\hline \multicolumn{7}{|l|}{ Residence, n (\%) } \\
\hline Urban & $\begin{array}{c}318 \\
(11.3 \%)\end{array}$ & -- & $48(8.6 \%)$ & -- & $\begin{array}{c}270 \\
(12.0 \%)\end{array}$ & -- \\
\hline Suburb & $\begin{array}{c}873 \\
(31.1 \%)\end{array}$ & ${ }_{--}^{*}$ & $\begin{array}{c}220 \\
(39.2 \%)\end{array}$ & $-{ }^{*}$ & $\begin{array}{c}653 \\
(29.0 \%)\end{array}$ & $-^{*}$ \\
\hline Rural & $\begin{array}{c}1621 \\
(57.6 \%)\end{array}$ & $-^{*}$ & $\begin{array}{c}293 \\
(52.2 \%)\end{array}$ & - $*$ & $\begin{array}{c}1328 \\
(59.0 \%)\end{array}$ & -- \\
\hline Han Ethnicity, n (\%) & $\begin{array}{c}2623 \\
(93.3 \%)\end{array}$ & -- $^{*}$ & $\begin{array}{c}502 \\
(89.5 \%)\end{array}$ & $-^{*}$ & $\begin{array}{c}2121 \\
(94.2 \%)\end{array}$ & $-^{*}$ \\
\hline \multicolumn{7}{|l|}{$\begin{array}{l}\text { Occupation before retirement, } \mathrm{n} \\
(\%)\end{array}$} \\
\hline Agriculture & $\begin{array}{c}1115 \\
(39.6 \%)\end{array}$ & ${ }_{--}^{*}$ & $\begin{array}{c}241 \\
(43.0 \%)\end{array}$ & $-^{*}$ & $\begin{array}{c}874 \\
(38.8 \%)\end{array}$ & -- \\
\hline Employee & $\begin{array}{c}486 \\
(17.3 \%) \\
\end{array}$ & -- & $\begin{array}{c}75 \\
(13.3 \%) \\
\end{array}$ & -- & $\begin{array}{c}411 \\
(18.3 \%) \\
\end{array}$ & $-^{*}$ \\
\hline Other & $\begin{array}{c}1211 \\
(43.1 \%)\end{array}$ & - ${ }^{*}$ & $\begin{array}{c}245 \\
(43.7 \%)\end{array}$ & ${ }^{*}{ }^{*}$ & $\begin{array}{c}966 \\
(42.9 \%)\end{array}$ & -- \\
\hline MMSE score & $25.8(5.2)$ & $24.0(7.1)$ & $26.4(4.8)$ & $24.3(6.8)$ & $25.7(5.3)$ & $23.9(7.2)$ \\
\hline $\mathrm{PM}_{2.5}, \mu \mathrm{g} / \mathrm{m}^{3}$ & $58.2(14.8)$ & $41.5(11.3)$ & $\begin{array}{c}42.2 \\
(14.3) \\
\end{array}$ & $31.3(8.4)$ & $62.1(12.0)$ & $44.1(10.4)$ \\
\hline $\mathrm{PM}_{10}, \mu \mathrm{g} / \mathrm{m}^{3}$ & $84.2(25.7)$ & $70.2(22.0)$ & $\begin{array}{c}54.6 \\
(15.5)\end{array}$ & $47.0(10.8)$ & $91.6(22.2)$ & $76.0(20.2)$ \\
\hline $\mathrm{SO}_{2}, \mu \mathrm{g} / \mathrm{m}^{3}$ & $25.5(11.2)$ & $12.8(3.8)$ & $14.6(8.0)$ & $10.6(3.4)$ & $28.2(10.2)$ & $13.4(3.6)$ \\
\hline $\mathrm{NO}_{2}, \mu \mathrm{g} / \mathrm{m}^{3}$ & $26.6(11.7)$ & $25.5(9.6)$ & $14.8(7.9)$ & $14.6(6.1)$ & $29.5(10.6)$ & $28.2(8.3)$ \\
\hline $\mathrm{CO}, \mu \mathrm{g} / \mathrm{m}^{3}$ & $0.86(0.30)$ & $0.78(0.17)$ & $\begin{array}{c}0.61 \\
(0.14)\end{array}$ & $0.66(0.14)$ & $\begin{array}{l}0.919 \\
(0.30)\end{array}$ & $0.81(0.17)$ \\
\hline Max 8-hr $\mathrm{O}_{3}, \mu \mathrm{g} / \mathrm{m}^{3}$ & $85.5(11.2)$ & $94.5(12.4)$ & $\begin{array}{c}79.7 \\
(15.7)\end{array}$ & $80.3(5.5)$ & $86.9(9.2)$ & $98.0(11.0)$ \\
\hline Temperature, ${ }^{\circ} \mathrm{C}$ & $24.6(5.1)$ & $24.4(4.7)$ & $24.9(5.8)$ & $24.7(6.1)$ & $24.3(5.4)$ & $24.2(3.8)$ \\
\hline $\begin{array}{l}\text { Married and lived with spouse, } \\
\mathrm{n}(\%)\end{array}$ & $\begin{array}{c}1376 \\
(48.9 \%)\end{array}$ & $\begin{array}{c}1580 \\
(56.2 \%)\end{array}$ & $\begin{array}{c}321 \\
(57.2 \%)\end{array}$ & $\begin{array}{c}344 \\
(61.3 \%)\end{array}$ & $\begin{array}{c}1055 \\
(46.9 \%)\end{array}$ & $\begin{array}{c}1236 \\
(54.9 \%)\end{array}$ \\
\hline Regular physical activity, n (\%) & $\begin{array}{c}884 \\
(31.4 \%)\end{array}$ & $\begin{array}{c}809 \\
(28.8 \%)\end{array}$ & $\begin{array}{c}153 \\
(27.3 \%)\end{array}$ & $\begin{array}{c}154 \\
(27.5 \%)\end{array}$ & $\begin{array}{c}731 \\
(32.5 \%)\end{array}$ & $\begin{array}{c}655 \\
(29.1 \%)\end{array}$ \\
\hline Current alcohol drinker, n (\%) & $\begin{array}{c}498 \\
(17.7 \%)\end{array}$ & $\begin{array}{c}430 \\
(15.3 \%)\end{array}$ & $\begin{array}{c}97 \\
(17.3 \%)\end{array}$ & $87(15.5 \%)$ & $\begin{array}{c}401 \\
(17.8 \%)\end{array}$ & $\begin{array}{c}343 \\
(15.2 \%)\end{array}$ \\
\hline Current smoker, n (\%) & $\begin{array}{c}518 \\
(18.4 \%)\end{array}$ & $450(16 \%)$ & $\begin{array}{c}106 \\
(18.9 \%)\end{array}$ & $92(16.4 \%)$ & $\begin{array}{c}412 \\
(18.3 \%)\end{array}$ & $\begin{array}{c}358 \\
(15.9 \%)\end{array}$ \\
\hline \multicolumn{7}{|l|}{ Fruit intake, n (\%) } \\
\hline \multicolumn{7}{|l|}{\begin{tabular}{|l|l} 
& Very often
\end{tabular}} \\
\hline Often & $\begin{array}{c}835 \\
(29.7 \%)\end{array}$ & $\begin{array}{c}720 \\
(25.6 \%)\end{array}$ & $\begin{array}{c}161 \\
(28.7 \%)\end{array}$ & $\begin{array}{c}176 \\
(31.4 \%)\end{array}$ & $\begin{array}{c}674 \\
(29.9 \%)\end{array}$ & $\begin{array}{c}590 \\
(26.2 \%)\end{array}$ \\
\hline Sometime & $\begin{array}{c}950 \\
(33.8 \%)\end{array}$ & $\begin{array}{c}904 \\
(32.1 \%)\end{array}$ & $\begin{array}{c}197 \\
(35.1 \%)\end{array}$ & $\begin{array}{c}194 \\
(34.6 \%)\end{array}$ & $\begin{array}{c}753 \\
(33.4 \%)\end{array}$ & $\begin{array}{c}714 \\
(31.7 \%)\end{array}$ \\
\hline Rare & $\begin{array}{c}619 \\
(21.8 \%)\end{array}$ & $\begin{array}{c}674 \\
(25.1 \%)\end{array}$ & $\begin{array}{c}128 \\
(22.8 \%)\end{array}$ & $\begin{array}{c}113 \\
(20.1 \%)\end{array}$ & $\begin{array}{c}491 \\
(21.8 \%)\end{array}$ & $\begin{array}{c}568 \\
(25.1 \%)\end{array}$ \\
\hline \multicolumn{7}{|l|}{ Vegetable intake, n (\%) } \\
\hline Very often & $\begin{array}{c}1701 \\
(60.5 \%)\end{array}$ & $\begin{array}{c}1691 \\
(60.1 \%)\end{array}$ & $\begin{array}{c}345 \\
(61.5 \%)\end{array}$ & $\begin{array}{c}377 \\
(67.2 \%)\end{array}$ & $\begin{array}{c}1356 \\
(60.2 \%)\end{array}$ & $\begin{array}{c}1314 \\
(58.4 \%)\end{array}$ \\
\hline
\end{tabular}




\begin{tabular}{|c|c|c|c|c|c|c|c|}
\hline & Often & $\begin{array}{c}884 \\
(31.4 \%)\end{array}$ & $\begin{array}{c}854 \\
(30.4 \%)\end{array}$ & $\begin{array}{c}144 \\
(25.7 \%)\end{array}$ & $\begin{array}{c}121 \\
(21.6 \%)\end{array}$ & $\begin{array}{c}740 \\
(32.9 \%)\end{array}$ & $\begin{array}{c}733 \\
(32.6 \%)\end{array}$ \\
\hline & Sometime & $174(6.2 \%)$ & $200(7.1 \%)$ & 50 (8.9\%) & 41 (7.3\%) & $124(5.5 \%)$ & $159(7.1 \%)$ \\
\hline & Rare & $53(1.9 \%)$ & $67(2.4 \%)$ & $22(3.9 \%)$ & $22(3.9 \%)$ & $31(1.4 \%)$ & $45(2.0 \%)$ \\
\hline \multicolumn{8}{|c|}{ Water quality, n (\%) } \\
\hline & Tap water & $\begin{array}{c}1896 \\
(67.4 \%)\end{array}$ & $\begin{array}{c}2091 \\
(74.4 \%)\end{array}$ & $\begin{array}{c}325 \\
(57.9 \%)\end{array}$ & $\begin{array}{c}364 \\
(64.9 \%)\end{array}$ & $\begin{array}{c}1571 \\
(69.8 \%)\end{array}$ & $\begin{array}{c}1727 \\
(76.7 \%)\end{array}$ \\
\hline & $\begin{array}{l}\text { Natural } \\
\text { water }\end{array}$ & $226(8.1 \%)$ & $208(7.4 \%)$ & $\begin{array}{c}83 \\
(14.8 \%)\end{array}$ & $44(7.8 \%)$ & $143(6.4 \%)$ & $164(7.3 \%)$ \\
\hline & Well water & $\begin{array}{c}690 \\
(24.5 \%)\end{array}$ & $\begin{array}{c}513 \\
(18.2 \%)\end{array}$ & $\begin{array}{c}153 \\
(27.8 \%)\end{array}$ & $\begin{array}{c}153 \\
(27.2 \%)\end{array}$ & $\begin{array}{c}537 \\
(23.8 \%)\end{array}$ & $\begin{array}{c}360 \\
(16.0 \%)\end{array}$ \\
\hline \multicolumn{8}{|c|}{ Living condition, $\mathrm{n}(\%)$} \\
\hline & $\begin{array}{l}\text { Family } \\
\text { members }\end{array}$ & $\begin{array}{c}2159 \\
(76.8 \%) \\
\end{array}$ & $\begin{array}{c}2159 \\
(76.8 \%) \\
\end{array}$ & $\begin{array}{c}421 \\
(75.0 \%) \\
\end{array}$ & $\begin{array}{c}451 \\
(80.4 \%) \\
\end{array}$ & $\begin{array}{c}1738 \\
(77.2 \%) \\
\end{array}$ & $\begin{array}{c}1708 \\
(75.9 \%) \\
\end{array}$ \\
\hline & Living alone & $\begin{array}{c}622 \\
(22.2 \%)\end{array}$ & $\begin{array}{c}607 \\
(21.6 \%)\end{array}$ & $\begin{array}{c}133 \\
(23.8 \%)\end{array}$ & $90(16.0 \%)$ & $\begin{array}{c}489 \\
(21.7 \%)\end{array}$ & $\begin{array}{c}503 \\
(22.3 \%)\end{array}$ \\
\hline & Elders' home & $31(1.1 \%)$ & $46(1.6 \%)$ & $7(1.2 \%)$ & $6(1.1 \%)$ & $24(1.1 \%)$ & $40(1.8 \%)$ \\
\hline \multicolumn{8}{|c|}{ Income source, $\mathrm{n}(\%)$} \\
\hline Family support & $1352(48.1 \%)$ & $\begin{array}{c}1422 \\
(50.6 \%)\end{array}$ & $\begin{array}{c}317 \\
(56.5 \%)\end{array}$ & $\begin{array}{c}314 \\
(56.0 \%)\end{array}$ & $\begin{array}{c}1035 \\
(46.0 \%)\end{array}$ & $\begin{array}{c}1108 \\
(49.2 \%)\end{array}$ & \\
\hline $\begin{array}{l}\text { Retirement } \\
\text { pension }\end{array}$ & $608(21.6 \%)$ & $\begin{array}{c}620 \\
(22.1 \%)\end{array}$ & $\begin{array}{c}113 \\
(20.1 \%)\end{array}$ & $\begin{array}{c}113 \\
(20.1 \%)\end{array}$ & $\begin{array}{c}495 \\
(22.0 \%)\end{array}$ & $\begin{array}{c}507 \\
(22.5 \%) \\
\end{array}$ & \\
\hline Social insurance & $235(8.4 \%)$ & $244(8.7 \%)$ & $71(12.7 \%)$ & $\begin{array}{c}59 \\
(10.5 \%) \\
\end{array}$ & $164(7.3 \%)$ & $185(8.2 \%)$ & \\
\hline $\begin{array}{l}\text { Working } \\
\text { payment }\end{array}$ & $413(14.7 \%)$ & $264(9.4 \%)$ & $46(8.2 \%)$ & $26(4.6 \%)$ & $\begin{array}{c}367 \\
(16.3 \%)\end{array}$ & $\begin{array}{c}238 \\
(10.6 \%)\end{array}$ & \\
\hline Other & $204(7.3 \%)$ & $262(9.3 \%)$ & $14(2.5 \%)$ & $49(8.7 \%)$ & $190(8.4 \%)$ & $213(9.5 \%)$ & \\
\hline
\end{tabular}

Data are mean (standard deviation), unless otherwise specified.

* Data were the same as in 2014.

Max 8-hr $\mathrm{O}_{3}$, max 8-hour daily average ozone measurements.

\section{Figures}

\begin{tabular}{|c|c|c|c|}
\hline \multicolumn{2}{|l|}{ PM reduction targets } & $\begin{array}{c}\text { Effect estimate }(95 \% \mathrm{CI}) \text {, } \\
\text { MMSE score }\end{array}$ & $P$ value \\
\hline \multicolumn{4}{|l|}{ Cut-off $\geq 5 \%$} \\
\hline Model 1 & $\longrightarrow$ & $1.36(0.47,2.25)$ & 0.003 \\
\hline Model 2 & $\longrightarrow$ & $2.38(1.25,3.50)$ & $<0.001$ \\
\hline Model 3 & $\longrightarrow$ & $2.35(1.22,3.48)$ & $<0.001$ \\
\hline Model 4 & & $2.45(1.32,3.57)$ & $<0.001$ \\
\hline Cut-off $\geq 10 \%$ & $?$ & $1.39(0.52,2.27)$ & 0.002 \\
\hline Cut-off $\geq 12 \%$ & - & $1.27(0.45,2.09)$ & 0.003 \\
\hline Cut-off $\geq 15 \%$ & $\therefore$ & $1.07(0.22,1.92)$ & 0.014 \\
\hline
\end{tabular}


Figure 1

The estimated effect of the CCAA on changes in MMSE score by difference-in-differences models. Note: CCAA, China's Clean Air Act; MMSE, Mini-Mental State Examination. Model 1: adjusted for inverse probability weighting from baseline and offset of MMSE trend; Model 2: further adjusted survey month based on Model 1; Model 3: further adjusted marital status, alcohol drinking, smoking, and physical activity based on Model 2; Model 4: further adjusted intake of fruit and vegetables, water quality, living condition, and income source. Effect estimates were also provided using different cut-offs of the annual PM reduction targets ( $\geq 10 \%, \geq 12 \%$, and $\geq 15 \%$ ) other than $\geq 5 \%$.
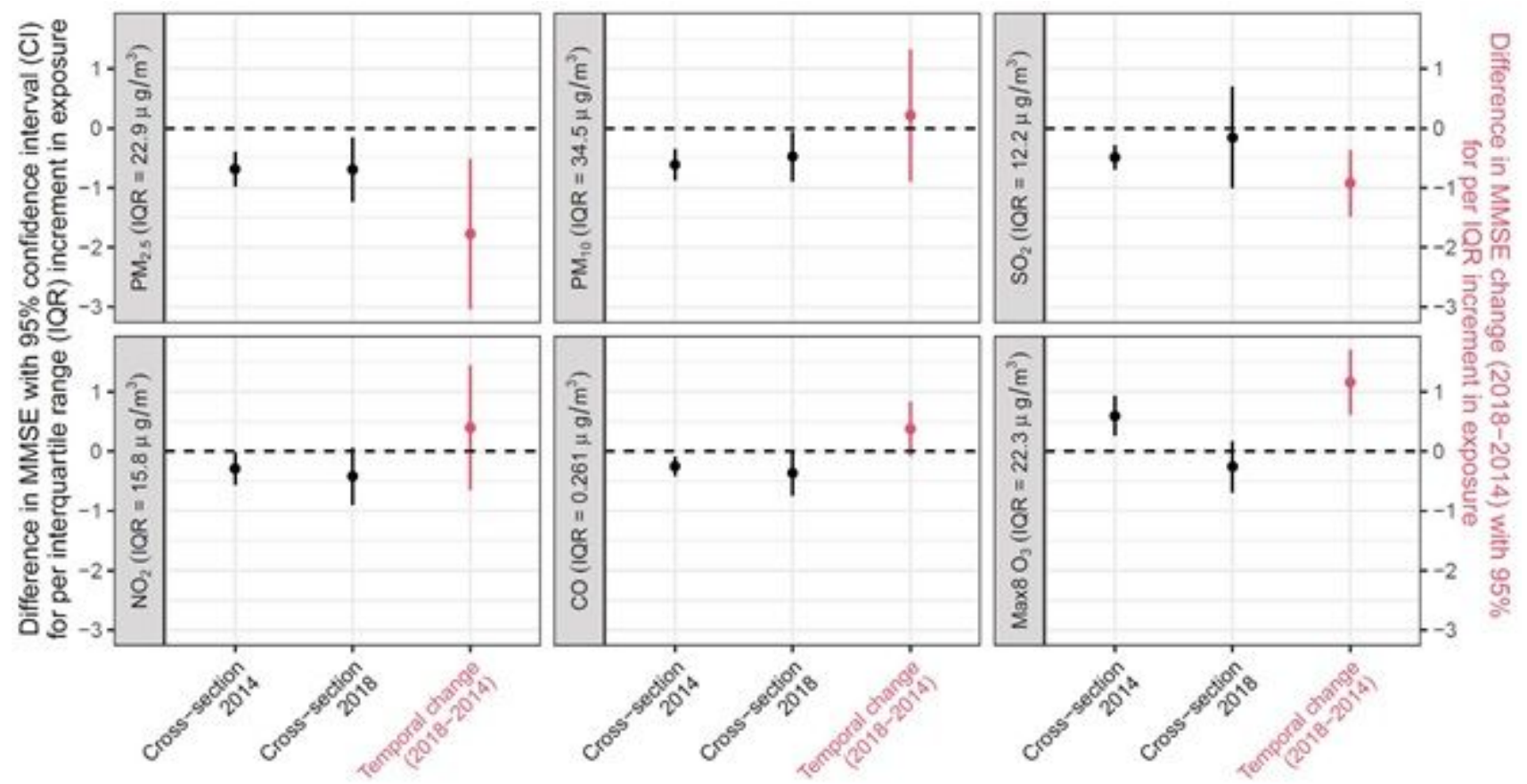

Air pollution exposure

\section{Figure 2}

Changes in MMSE score associated with an interquartile range (IQR) increase in pollutant exposure. In the cross-sectional analyses, MMSE scores were regressed against pollutant exposure across all participants in each of the two waves. In the temporal (2018 vs. 2014) change analysis, within-person temporal changes in MMSE score were regressed against temporal changes in pollutant exposure. In all models, selected covariates were adjusted for. 

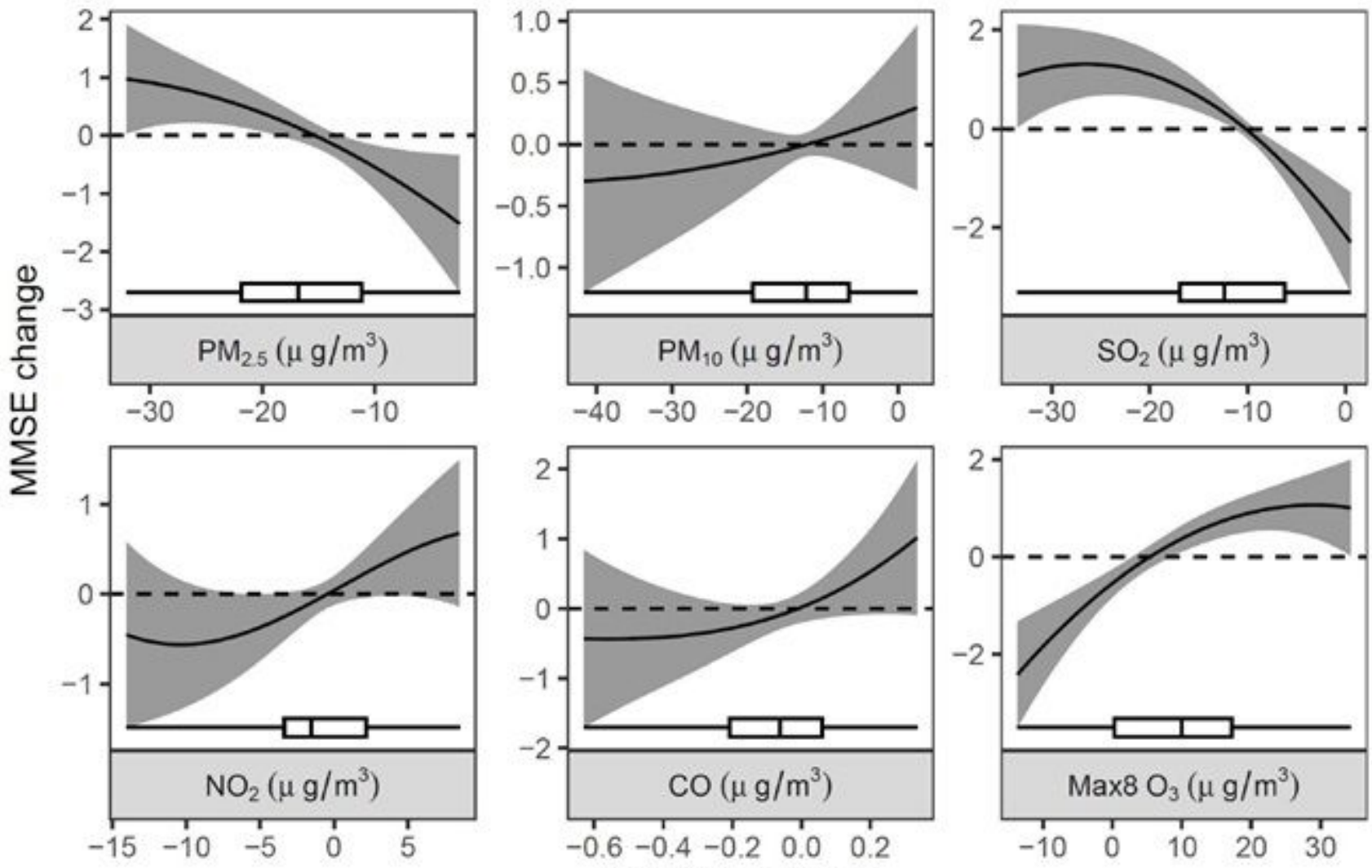

Exposure change

Figure 3

The nonlinear estimates for the associations between air pollution changes and MMSE score changes by difference-in-differences models. Solid line: fitted estimates of the associations between air pollution changes and MMSE score changes; shaded areas: 95\% confidence intervals. MMSE: Mini-Mental State Examination. 


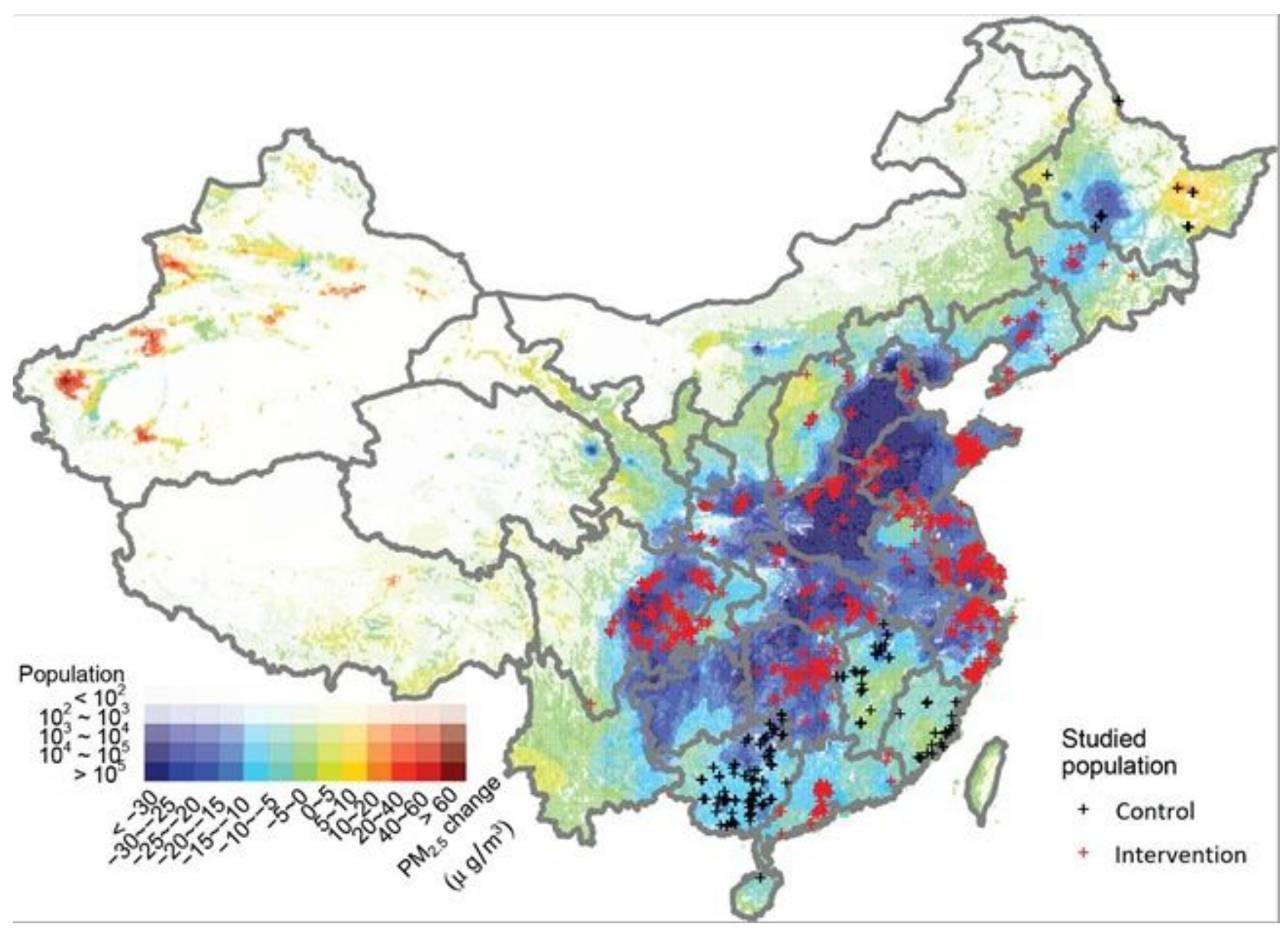

\section{Figure 4}

Map of study areas with changes in annual mean of PM2.5 from 2014 to 2017 . The study participants were designated into control (black dot) and intervention (red dot) groups, according to the preestablished target of reduced concentration of fine particulate matters (PM2.5) after 2014. Intervention group: target of annual PM2.5 reduction $\geq 5 \%$ under CCAA; Control group: without reduction target under CCAA. In the present analysis, we used the data collected in the 7th (2014) and 8th (2018) waves of the CLHLS. These two waves spanned an ideal time period for exploring a potentially causal relationship between cognitive function and air pollution using a quasi-experimental framework, as PM2.5 exposure reduced substantially in a fraction of CLHLS participants while economic, socio-behavioral, and other factors remained relatively stable over these 4 years. The analytical samples comprised individuals who (1) were free of dementia, stroke, and bed-ridden status at the 2014 wave, (2) had cognitive function examinations at the both waves, (3) provided primary residential addresses, allowing for assessing air pollution exposures, (4) were non-movers during follow-up, and (5) had no missing data for any of the confounding variables. Our final sample size consisted of 2812 participants (Supplementary Methods, Flow chart). CLHLS was approved by the Biomedical Ethics Committee, Peking University (IRB0000105213074). All participants provided written informed consent prior to participation. Note: The designations employed and the presentation of the material on this map do not imply the expression of any opinion whatsoever on the part of Research Square concerning the legal status of any country, territory, city or 
area or of its authorities, or concerning the delimitation of its frontiers or boundaries. This map has been provided by the authors.

\section{Supplementary Files}

This is a list of supplementary files associated with this preprint. Click to download.

- 03SupplementaryInformation.docx 